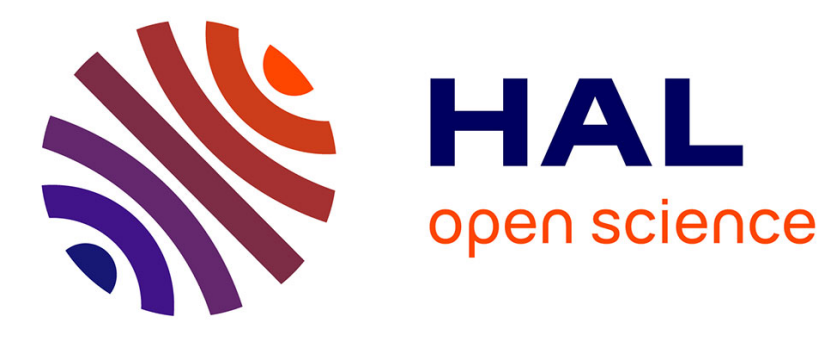

\title{
D1-Input-to-State Stability of a Time-Varying Nonhomogeneous Diffusive Equation Subject to Boundary Disturbances
}

Federico Bribiesca Argomedo, Emmanuel Witrant, Christophe Prieur

\section{To cite this version:}

Federico Bribiesca Argomedo, Emmanuel Witrant, Christophe Prieur. D1-Input-to-State Stability of a Time-Varying Nonhomogeneous Diffusive Equation Subject to Boundary Disturbances. ACC 2012 - American Control Conference, Jun 2012, Montréal, Canada. pp.2978-2983, 10.1109/ACC.2012.6315060 . hal-00676534

\section{HAL Id: hal-00676534 \\ https://hal.science/hal-00676534}

Submitted on 2 Apr 2019

HAL is a multi-disciplinary open access archive for the deposit and dissemination of scientific research documents, whether they are published or not. The documents may come from teaching and research institutions in France or abroad, or from public or private research centers.
L'archive ouverte pluridisciplinaire HAL, est destinée au dépôt et à la diffusion de documents scientifiques de niveau recherche, publiés ou non, émanant des établissements d'enseignement et de recherche français ou étrangers, des laboratoires publics ou privés. 


\title{
$D^{1}$-Input-to-State Stability of a Time-Varying Nonhomogeneous Diffusive Equation Subject to Boundary Disturbances
}

\author{
Federico Bribiesca Argomedo, Emmanuel Witrant and Christophe Prieur
}

\begin{abstract}
D^{1}$-Input-to-state stability ( $D^{1}$ ISS) of a diffusive equation with Dirichlet boundary conditions is shown, in the $L^{2}$-norm, with respect to boundary disturbances. In particular, the spatially distributed diffusion coefficients are allowed to be time-varying within a given set, without imposing any constraints on their rate of variation. Based on a strict Lyapunov function for the system with homogeneous boundary conditions, $D^{1}$ ISS inequalities are derived for the disturbed equation. A heuristic method used to numerically compute weighting functions is discussed. Numerical simulations are presented and discussed to illustrate the implementation of the theoretical results.
\end{abstract}

\section{INTRODUCTION}

Parabolic partial differential equations (PDEs) are used to model a wide array of physical phenomena. Within this class of equations, diffusion or diffusive equations are commonly encountered. For most physical systems in which diffusive effects are present, diffusivity coefficients can be approximated as being constant throughout the domain of interest. However, in particular when dealing with nonhomogeneous or anisotropic (direction-dependent) media, the use of distributed coefficients is required. The extension of results obtained with constant-coefficients to these cases is not always easy to tackle and can be particularly complicated when the coefficients are time-varying.

Input-to-state stability (ISS) analysis for nonlinear finitedimensional systems has been a long standing research topic and thorough reviews of such results can be found (see for example [17] and [8]). Nevertheless, ISS properties are not restricted to finite-dimensional systems. Some particularly interesting examples in an infinite-dimensional framework are: [7], where a frequency-domain approach is used to guarantee ISS properties; [12], where a strict Lyapunov function is constructed for semilinear parabolic PDEs; and [14], where a strict Lyapunov function is used for timevarying hyperbolic PDEs. The notion of $\mathrm{D}^{k} \mathrm{ISS}$, as presented in [17], is similar to that of ISS. In this case however, the norm of the state is bounded not only by the initial conditions

The authors are with, Université de Grenoble / Université Joseph Fourier / CNRS, GIPSA-lab UMR 5216 Grenoble, France. E-mail: federico.bribiescaargomedo@gipsa-lab.inpg.fr. This work was carried out within the framework of the European Fusion Development Agreement and the French Research Federation for Fusion Studies. It is supported by the European Communities under the contract of Association between EURATOM and CEA. The views and opinions expressed herein do not necessarily reflect those of the European Commission. The research leading to these results has also received funding from the European Union Seventh Framework Programme [FP7/2007-2013] under grant agreement $\mathrm{n}^{\circ} 257462$ HYCON2 Network of excellence and from the BQR Grenoble-INP. and the norm of the input signal, but also by the norm of the first $k$ time-derivatives of this input signal.

The use of Lyapunov functions to study the solutions or properties of infinite-dimensional systems is not new, see for instance [1], but it is still an active research topic. Other interesting results involving Lyapunov functions applied to parabolic equations can be found in [3], where a Lyapunov approach is used to prove the existence of a global solution to the heat equation; [10], where Lyapunov functions are used to analyze the regularity and well-posedness of Burgers' equation with a backstepping boundary control; [9], where a Lyapunov function is used to analyze the heat equation with unknown destabilizing parameters and its control extensions in [15] and [16]. Other results not involving parabolic equations are for example [5], where a Lyapunov function is used for the stabilization of a rotating beam; or more recently [6], where the construction of stabilizing boundary controls for a system of conservation laws is tackled using a Lyapunov function. In [4] a Lyapunov function is used for the stability analysis of nonlinear hyperbolic systems.

There are two main contributions in this paper using the strict Lyapunov function constructed in [2]. The first contribution is to set sufficient conditions for $\mathrm{D}^{1} \mathrm{ISS}$, in the $L^{2}$-norm, with respect to boundary disturbances in a timevarying nonhomogeneous diffusive equation with rapidly (yet smoothly) time-varying coefficients. The second contribution is providing a heuristic method for numerically computing adequate weighting functions in order to apply the theoretical results. Strict Lyapunov functions are chosen since they provide a natural framework for dealing with robustness issues and possibly considering some nonlinearities in the system behaviour.

This article is organized as follows. In Section II, the diffusive equation and boundary disturbances under consideration are presented. In Section III the main result of the paper, a $\mathrm{D}^{1}$ ISS inequality with respect to boundary disturbances, is obtained and combined with the sufficient condition (14) derived in [2] to find a strict Lyapunov function. In Section $\mathrm{IV}$, a heuristic method to find a suitable weighting function for exponential-like diffusivity coefficients is presented. In Section V, a weighting function is provided and numerical simulations are presented for the system with and without boundary disturbances.

\section{Notation}

Throughout this paper the following notations are used: $\mathbb{R}^{+}=(0,+\infty)$; given a function $\xi:(r, t) \mapsto \xi(r, t)$, its partial derivatives with respect to $r$ and $t$ are denoted as $\xi_{r}$ 
and $\xi_{t}$, respectively; given a function of time $\Xi: t \mapsto \Xi(t)$, the derivative of $\Xi$ with respect to time is denoted $\dot{\Xi}$; given an almost everywhere (a.e.) twice-differentiable function $g$ : $r \mapsto g(r)$ (i.e. a function having a second derivative equal to a piecewise continuous function except, perhaps, on a zero-measure set), $g^{\prime}(r)$ represents an absolutely continuous function equal a.e. to the first derivative of $g$ with respect to $r$. Analogously, $g^{\prime \prime}(r)$ represents a piecewise continuous function equal a.e. to the second derivative of $g$ with respect to $r$. For a function $g \in L^{2}([0,1])$, its $L^{2}$ norm will be noted $\|g\|_{L^{2}} \doteq\left(\int_{0}^{1} g^{2}(\rho) d \rho\right)^{\frac{1}{2}}$.

\section{Model And Problem Statement}

Consider the following two-dimensional equation with symmetric coefficients (defined in $\Omega \doteq$ $\left.\left\{x=\left(x_{1}, x_{2}\right) \in \mathbb{R}^{2} \mid x_{1}^{2}+x_{2}^{2}<1\right\}\right)$ expressed in Cartesian coordinates:

$$
\zeta_{t}(x, t)=\eta(x, t) \Delta \zeta(x, t), \forall(x, t) \in \Omega \times[0, T)
$$

where $\Delta$ is the Laplacian operator. The symmetric, disturbed boundary condition is:

$$
\zeta_{\nu}(x, t)=\varepsilon(t), \forall(x, t) \in \partial \Omega \times[0, T)
$$

where $\zeta_{\nu}$ is the derivative of $\zeta$ in the outward normal direction to $\partial \Omega$, and symmetric initial condition belonging to $C^{1}(\bar{\Omega})$ :

$$
\zeta(x, 0)=\zeta_{0}(x), \forall x \in \bar{\Omega}
$$

Under the revolution symmetry condition, system (1)-(3) can be reduced to a one-dimensional representation, in polar coordinates. Throughout this article, we are interested in the evolution of the variable $z \doteq \nabla \zeta \cdot \vec{\rho}$ (where $\vec{\rho}$ is the unit vector in the radial direction and $\nabla$ the gradient operator), given by:

$$
z_{t}=\left[\frac{\eta(r, t)}{r}[r z]_{r}\right]_{r}, \forall(r, t) \in(0,1) \times[0, T)
$$

with disturbed Dirichlet boundary condition:

$$
\begin{aligned}
& z(0, t)=0, \quad \forall t \in[0, T) \\
& z(1, t)=\varepsilon(t), \forall t \in[0, T)
\end{aligned}
$$

where the condition at the center is given by the symmetry and regularity of the solutions, and with initial condition:

$$
z(r, 0)=z_{0}(r), \forall r \in[0,1]
$$

where $z_{0} \doteq \nabla \zeta_{0} \cdot \vec{\rho}$.

The following assumption is used for the analysis of the well-posedness of the problem:

A1: $\eta$ is positive and belongs to $C^{\infty}(\bar{\Omega} \times[0, T])$. $\varepsilon$ belongs to $C^{\infty}([0, T])$.

Based on Theorem 6.2 in [13] (page 228), and using the same procedure as in Section II of [2] we have that:

Proposition 1: Under assumption A1, for every $z_{0}$ : $[0,1] \rightarrow \mathbb{R}$ in $L^{p}([0,1]), 1<p<\infty$, the evolution equations (4)-(6) have a unique solution $z$ in $C^{\infty}([0,1] \times(0, T))$.
Remark 1: The regularity conditions in Al can be relaxed if the solution $z$ is also allowed to be less regular, applying for example Theorem 5.1.21 in [11], but it is beyond the scope of this article. Hereafter, sufficiently regular solutions to (4)-(6) are assumed to exist.

Our goal is to solve the following problem:

Problem 1: Given a bounded time-varying disturbance signal $t \mapsto \varepsilon(t)$ with bounded derivative $\dot{\varepsilon}(t)$, find some bounds for the $L^{2}$-norm of the solution $z$ of (4)-(6).

\section{STRICT LyAPUNOV FUNCTION AND SUFFICIENT CONDITIONS FOR D ${ }^{1}$-INPUT-TO-STATE STABILITY}

Consider the following candidate Lyapunov function, for $z \in L^{2}([0,1])$ :

$$
V(z) \doteq \frac{1}{2} \int_{0}^{1} f(r) z^{2} d r
$$

where $f:[0,1] \rightarrow \mathbb{R}^{+}$is an a.e. twice-differentiable positive function with piecewise-continuous second derivative.

Following [17] and other references, $V$ is said to be a strict Lyapunov function for the undisturbed version of system (4)(6) if, when setting $\varepsilon(t)=0$ for all $t \in[0, T)$, there exists some positive constant $\alpha$ such that, for every initial condition $z_{0}$ as defined in (6):

$$
\dot{V} \leq-\alpha V(z(\cdot, t)), \forall t \in[0, T)
$$

where $\dot{V}$ stands for the time derivative of $V$ along the trajectory of the undisturbed system stemming from $z_{0}$.

Hereafter, we define for $g \in L^{2}([0,1])$ its weighted $L^{2}$ norm as $\|g\|_{f} \doteq(V(g))^{\frac{1}{2}}$.

A useful technical assumption is introduced:

A2: There exists a weighting function $f$ as defined in (7) such that $V$ is a strict Lyapunov function for system (4)-(6) if $\varepsilon(t)=0$ for all $t \in[0, T)$.

The next theorem constitutes the main contribution of this article:

Theorem 2: Under Assumptions A1 and A2, the following inequality is satisfied, for all $t_{0} \in[0, T)$, by the state of the disturbed system (4)-(6):

$$
\begin{aligned}
\|z(\cdot, t)\|_{L^{2}} \leq & c e^{-\frac{\alpha}{2}\left(t-t_{0}\right)}\left[\left\|z\left(\cdot, t_{0}\right)\right\|_{L^{2}}+\frac{1}{\sqrt{3}}\left|\varepsilon\left(t_{0}\right)\right|\right] \\
& +c \int_{t_{0}}^{t} e^{-\frac{\alpha}{2}(t-\tau)}\|\bar{\varepsilon}(\cdot, \tau)\|_{L^{2}} d \tau \\
& +\frac{c}{\sqrt{3}}|\varepsilon(t)|
\end{aligned}
$$

where $\bar{\varepsilon}(r, t) \doteq 2 \eta_{r}(r, t) \varepsilon(t)-r \dot{\varepsilon}(t)$, for all $(r, t) \in[0,1] \times$ $\left[t_{0}, T\right), c \doteq \sqrt{\frac{f_{\text {max }}}{f_{\min }}}$ and $f_{\text {min }} \doteq \min _{r \in[0,1]}\{f(r)\}, f_{\text {max }} \doteq$ $\max _{r \in[0,1]}\{f(r)\}$.

Proof: Consider an alternative definition of the state variable:

$$
\hat{z}(r, t) \doteq z(r, t)-r \varepsilon(t), \forall(r, t) \in[0,1] \times\left[t_{0}, T\right)
$$

Using (10) and its time derivative in (4), the evolution of the new state variable $\hat{z}$ is obtained as:

$$
\hat{z}_{t}=\left[\frac{\eta}{r}[r \hat{z}]_{r}\right]_{r}+2 \eta_{r} \varepsilon-r \dot{\varepsilon}, \forall(r, t) \in(0,1) \times\left[t_{0}, T\right)
$$


where $\left[\frac{\eta}{r}[r \hat{z}]_{r}\right]_{r}+2 \eta_{r} \varepsilon$ is equivalent to $z_{t}(r, t)$, with Dirichlet boundary conditions:

$$
\hat{z}(0, t)=\hat{z}(1, t)=0, \forall t \in\left[t_{0}, T\right)
$$

and initial condition:

$$
\hat{z}\left(r, t_{0}\right)=z\left(r, t_{0}\right)-r \varepsilon\left(t_{0}\right), \forall r \in(0,1)
$$

Consider the function $V$ defined in (7) with a weighting function satisfying Assumption A2, applied to the reformulated system (11)-(12):

$$
V(\hat{z}) \doteq \frac{1}{2} \int_{0}^{1} f(r) \hat{z}^{2} d r
$$

From the definition of $\hat{z}$ in (10), we compute for all $t \in$ $\left[t_{0}, T\right)$ :

$$
\begin{aligned}
\dot{V}= & \int_{0}^{1} f(r) \hat{z}\left[\frac{\eta}{r}[r \hat{z}]_{r}\right]_{r} d r+ \\
& 2 \int_{0}^{1} f(r) \hat{z} \eta_{r} \varepsilon d r- \\
& \int_{0}^{1} f(r) \hat{z} r \dot{\varepsilon} d r
\end{aligned}
$$

Using inequality (8) this implies:

$$
\begin{aligned}
\dot{V} \leq & -\alpha V(\hat{z})+ \\
& 2 \int_{0}^{1} f(r) \hat{z} \eta_{r} \varepsilon d r- \\
& \int_{0}^{1} f(r) \hat{z} r \dot{\varepsilon} d r
\end{aligned}
$$

The definition of $\bar{\varepsilon}(r, t)$ in Theorem 2, provides the compact form:

$$
\dot{V} \leq-\alpha V(\hat{z})+\int_{0}^{1} f(r) \hat{z} \bar{\varepsilon} d r, \forall t \in\left[t_{0}, T\right)
$$

where, by the boundedness of $\varepsilon(t)$ and $\dot{\varepsilon}(t)$ in Problem 1, $\bar{\varepsilon}(r, t)$ is uniformly bounded in $[0,1] \times\left[t_{0}, T\right)$.

The last equation implies that:

$$
\dot{V} \leq-\alpha V(\hat{z})+\int_{0}^{1}|f(r) \hat{z} \bar{\varepsilon}| d r, \forall t \in\left[t_{0}, T\right)
$$

Using the Cauchy-Schwarz inequality in (13), we have:

$$
\dot{V} \leq-\alpha V(\hat{z})+\|\sqrt{f(r)} \hat{z}\|_{L^{2}}\|\sqrt{f(r)} \bar{\varepsilon}\|_{L^{2}}
$$

which implies:

$$
\dot{V} \leq-\alpha V(\hat{z})+2\|\hat{z}\|_{f}\|\bar{\varepsilon}\|_{f}
$$

from which:

$$
\frac{d}{d t}\|\hat{z}\|_{f} \leq-\frac{\alpha}{2}\|\hat{z}\|_{f}+\|\bar{\varepsilon}\|_{f}
$$

We consequently get for all $t \in\left[t_{0}, T\right)$ :

$\|\hat{z}(\cdot, t)\|_{f} \leq e^{-\frac{\alpha}{2}\left(t-t_{0}\right)}\left\|\hat{z}\left(\cdot, t_{0}\right)\right\|_{f}+\int_{t_{0}}^{t} e^{-\frac{\alpha}{2}(t-\tau)}\|\bar{\varepsilon}(\cdot, \tau)\|_{f} d \tau$
Recalling (10) and after some rearrangements, this implies:

$$
\begin{aligned}
\|z(\cdot, t)\|_{f} \leq & e^{-\frac{\alpha}{2}\left(t-t_{0}\right)}\left[\left\|z\left(\cdot, t_{0}\right)\right\|_{f}+\left|\varepsilon\left(t_{0}\right)\right|\|r\|_{f}\right] \\
& +\int_{t_{0}}^{t} e^{-\frac{\alpha}{2}(t-\tau)}\|\bar{\varepsilon}(\cdot, \tau)\|_{f} d \tau \\
& +|\varepsilon(t)|\|r\|_{f}, \quad \forall t \in\left[t_{0}, T\right)
\end{aligned}
$$

Using the equivalence between the $L^{2}$ and $\|\cdot\|_{f}$ norms, and simply majorating and minorating $f$ by $f_{\max }$ and $f_{\min }$ respectively, the previous inequality implies (9) and completes the proof.

A simple application of Theorem 2 yields the following corollary:

Corollary 3: If there is a non-negative constant $t_{0}$ such that for all $t \geq t_{0}, \varepsilon$ is zero, the state of the system (4)-(6) converges exponentially fast to zero in the topology of the $L^{2}$-norm.

To give a sufficient condition for Assumption A2 to hold, it is useful to apply Theorem 3.2 from [2] as follows:

Proposition 4: If there exist $f$, as defined in (7), and a positive constant $\alpha$ such that, for all $(r, t) \in(0,1] \times[0, T)$ :

$$
f^{\prime \prime}(r) \eta+f^{\prime}(r)\left[\eta_{r}-\frac{\eta}{r}\right]+f(r)\left[\frac{\eta_{r}}{r}-\frac{\eta}{r^{2}}+\alpha\right] \leq 0
$$

then Assumption A2 holds.

Remark 2: Up to this point, no assumption on the shape or behaviour of $\eta$ has been made other than some regularity requirements. In the next section a particular shape of $\eta$, motivated by a physical application, is used to illustrate our result.

\section{Finding A Weighting Function}

The objective of this section is to propose a heuristic for numerically computing an adequate weighting function such that Assumption A2 holds. This is done by verifying the conditions of Proposition 4 for a particular set of diffusivity coefficients. In the rest of this article, the $\eta$ profile is assumed to be of the form:

$$
\eta(r, t)=a(t) e^{\int_{0}^{r} \phi(\xi, t) d \xi}, \forall(r, t) \in[0,1] \times[0, T)
$$

where $0<\underline{a} \leq a(t) \leq \bar{a}, \phi(r, t) \in \Phi=$ $\left\{\phi(r, t) \in \mathcal{C}^{\infty}([0,1] \times[0, T]) \mid \forall t \in[0, T], \phi(\cdot, t) \in \Lambda\right\}$, and $\Lambda=\left\{\lambda(r) \in \mathcal{C}^{\infty}([0,1]) \mid \forall r \in[0,1], \underline{\lambda} \leq \lambda(r) \leq \bar{\lambda}\right\}$.

This choice of profiles is physically motivated by the application of magnetic flux profile control in Tokamak plasmas, see Section V of [2] for a more detailed discussion.

Proposition 5: With $\eta$ defined as in (15), a sufficient condition to apply Proposition 4 is the existence of an a.e. twice-differentiable positive function $f:[0,1] \rightarrow \mathbb{R}^{+}$ with piecewise-continuous second derivative such that the following inequality is verified:

$$
f^{\prime \prime}(r)+f^{\prime}(r)\left[\lambda(r)-\frac{1}{r}\right]+f(r)\left[\frac{\lambda(r)}{r}-\frac{1}{r^{2}}+\epsilon\right] \leq 0
$$


Proof: multiplying (16) by $e^{\int_{0}^{r} \lambda(\xi) d \xi}$
$\epsilon \inf _{(r, \lambda) \in[0,1] \times \Lambda}\left\{\underline{a} e^{\int_{0}^{r} \lambda(\xi) d \xi}\right\}>0$

An a.e. twice-differentiable positive function with piecewise-continuous second derivative $f:[0,1] \rightarrow \mathbb{R}^{+}$ satisfies (16) if there exists $w(r, \lambda) \leq 0$ such that, for all $(r, \lambda) \in(0,1] \times \Lambda$ the following equation is verified:

$$
\left[\begin{array}{c}
f \\
f^{\prime}
\end{array}\right]^{\prime}=\left[\begin{array}{cc}
0 & 1 \\
\frac{1}{r^{2}}-\frac{\lambda(r)}{r}-\epsilon & \frac{1}{r}-\lambda(r)
\end{array}\right]\left[\begin{array}{l}
f \\
f^{\prime}
\end{array}\right]+\left[\begin{array}{l}
0 \\
1
\end{array}\right] w(r, \lambda)
$$

In order to avoid testing the condition for all $\lambda \in \Lambda$, the following result is used:

Proposition 6: Given an a.e. twice-differentiable positive function with piecewise-continuous second derivative $f$ : $[0,1] \rightarrow \mathbb{R}^{+}$, the following two conditions are equivalent:

$\mathrm{i}$ : there exists $w(r, \lambda) \leq 0$ such that (17) is verified for all $(r, \lambda) \in(0,1] \times \Lambda$;

ii: there exists $w_{2}(r) \leq 0$ such that the following equation is verified for all $r \in(0,1]$ :

$$
\left[\begin{array}{c}
f \\
f^{\prime}
\end{array}\right]^{\prime}=A(f, r)\left[\begin{array}{l}
f \\
f^{\prime}
\end{array}\right]+\left[\begin{array}{l}
0 \\
1
\end{array}\right] w_{2}(r)
$$

where:

$$
A(f, r)=\left\{\begin{array}{cc}
{\left[\begin{array}{cc}
0 & 1 \\
\frac{1}{r^{2}}-\frac{\lambda}{r}-\epsilon & \frac{1}{r}-\underline{\lambda}
\end{array}\right]} & \text { if } s w(f, r) \leq 0 \\
{\left[\begin{array}{cc}
0 & 1 \\
\frac{1}{r^{2}}-\frac{\bar{\lambda}}{r}-\epsilon & \frac{1}{r}-\bar{\lambda}
\end{array}\right]} & \text { if } s w(f, r)>0
\end{array}\right.
$$

where $s w(f, r) \doteq \frac{f(r)}{r}+f^{\prime}(r)$.

Proof: The proof stems from the fact that the left-hand side of (16), which is equivalent to (17), is linear in $\lambda(r)$ and and $\lambda$ is bounded by $\bar{\lambda}$ and $\underline{\lambda}$. It is easy to verify that the switching condition in matrix $A(f, r)$ corresponds to the sign of the coefficient of $\lambda(r)$ (at any given point $r$ ) in (16). Therefore, $w_{2}(r)=\sup _{\lambda \in \Lambda}\{w(r, \lambda)\}$ for all $r \in[0,1]$.

Remark 3: The easiest way to find a function $f$ that satisfies condition (18) is to fix some boundary conditions for $f$ and $f^{\prime}$, set $w_{2}(r)=0$ for all $r \in[0,1]$, and solve the resulting equation backwards from the values $f(1)$ and $f^{\prime}(1)$. Nevertheless, this yields solutions with a singularity at the origin, as can be seen in Figure 1 for $\underline{\lambda}=\bar{\lambda}=4$.

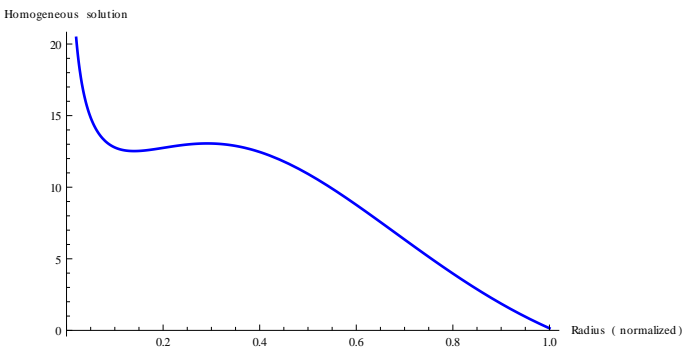

Fig. 1. Function $f$ obtained by numerically solving the homogeneous equation (17) for a single value of $\lambda=4$.

Since setting $w_{2}(r)=0$ does not suffice to find adequate weighting functions, a more structured approach is developed to tackle this problem. In order to compute a weight verifying condition ii of Proposition 6, boundary conditions are set at $r=1$ and the equation is solved backwards up to $r=0$ using Algorithm 1.

\section{Algorithm 1:}

1: Set numerical values for the boundary conditions at $r=$ $1, f(1)$ and $f^{\prime}(1)$, and for $\epsilon$.

2: Evaluate $\frac{f(r)}{r}+f^{\prime}(r)$ and fix the value of the dynamic matrix $A(f, r)$ accordingly, using (18).

3: Find a numerical solution going backwards until hitting a zero-crossing of $\frac{f(r)}{r}+f^{\prime}(r)$, setting $w_{2}(r)=0$, and verifying that $f(r)$ remains positive. Otherwise, change the boundary conditions or the value of $\epsilon$.

4: Use the values of $f(r)$ and $f^{\prime}(r)$ at the zero-crossings of $\frac{f(r)}{r}+f^{\prime}(r)$ as initial values for the next step in solving the equation, switching the dynamic matrix but keeping $w_{2}(r)=0$, always verifying that $f(r)$ remains positive and bounded.

5: Repeat 3-4 until either reaching $r=0$ or finding a point such that both elements in the lower row of the A matrix are positive, as well as $f$ and $f^{\prime}$, with $f(r)-r f^{\prime}(r)>$ 0 . If no such point exists before $r=0$, change the boundary conditions or the value of $\epsilon$ and start over.

6: If $r=0$ has not been reached yet, complete the solution by setting $w_{2}(r)$ to have $f^{\prime \prime}(r)=0$ for the remaining interval, in order to avoid singularities in the solution near zero.

Remark 4: Although this heuristic does not guarantee finding an adequate weighting function, it does provide a methodic framework to solve the nonlinear boundary value problem (18) and yields good results in practice, as shown in the next section.

The conclusion of this section is that Algorithm 1 gives a practical way for numerically testing condition ii in Proposition 6, which in turn, by Proposition 5 implies that the conditions of Proposition 4 are verified for $\eta$ defined as in (15). Proposition 4 being a sufficient condition for Assumption A2, Theorem 2 follows and Problem 1 is solved. It should be noted that this results also hold for any convex combination of functions $\eta$ satifying (15).

\section{NUMERICAL APPLICATION}

\section{A. Weighting Function}

We aim at finding an adequate weighting function for $a(t) \in[0.0093,0.0121], \underline{\lambda}=4$ and $\bar{\lambda}=7.3$ (see (15)). This would imply a $55 \%$ increase in the allowable range for $\eta(1, t)$ with respect to the objective set in [2] if we considered $\lambda$ constant in $r$. However, it should be noted that a much more general form of $\lambda(r)$ is being considered in this article.

The boundary conditions at $r=1$ were chosen as $f(1)=$ $0.15, f^{\prime}(1)=-15$, and a suitable weighting function was found for a maximum value of $\epsilon=5.3$. Given the values of the boundary conditions, the solution was obtained first using the dynamic matrix with $\underline{\lambda}$ and then switching dynamics at $r \approx 0.52$. For $r \in[0,0.015], f^{\prime \prime}$ was set to 0 using $w(r)$. The resulting weighting function, numerically computed using 


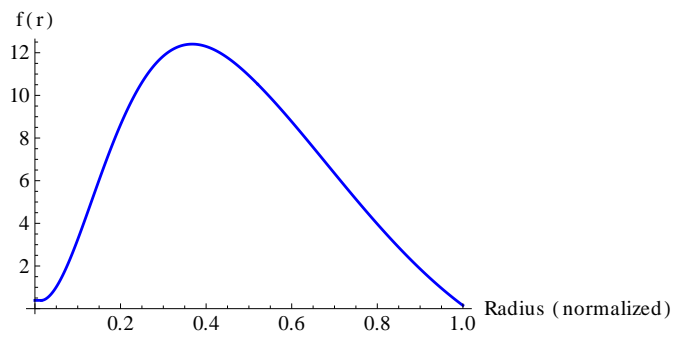

Fig. 2. Function $f$ obtained using the heuristic.

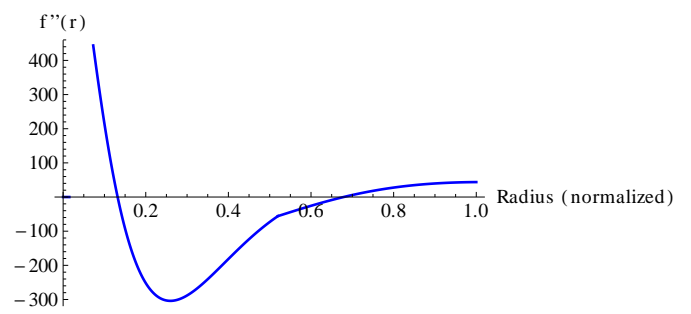

Fig. 3. Piecewise continuous second derivative of function $f$ obtained using the heuristic.

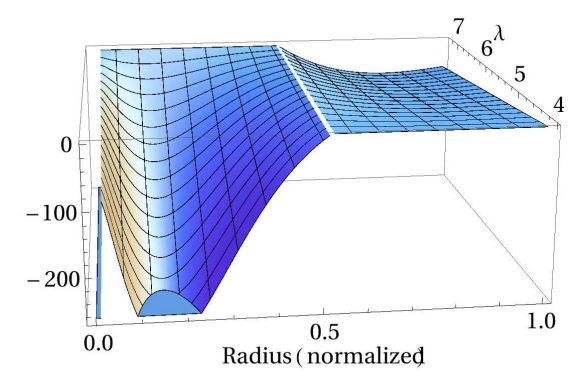

Fig. 4. Numerical test of the conditions of Proposition 5.

Mathematica ${ }^{\circledR}$, can be seen in Figure 2. The piecewise continuous and bounded second derivative of the weighting function is also shown in Figure 3. The maximum value of $f$ is $\sim 12.40$ and its minimum is 0.15 , which means that the constant $c$ used for the norm equivalence and in (9) has a value of $\sim 9.09$. Other functions with a much lower value of $c$ can be found, but usually there is a compromise between this constant and the guaranteed value for $\epsilon$.

In order to illustrate the fact that this function verifies the conditions of Proposition 5, the value of the left-hand side of inequality (16) was plotted for values of $(r, \lambda) \in$ $[0,1] \times[4,7.3]$ (with constant $\lambda$ throughout $r$ ). The result can be seen in Figure 4. It is interesting to note that for each value of $r$, the critical value of $\lambda$ in the inequality is the one used to compute the weighting function. For values of $r$ close to zero however, the slack variable $w$ is different from zero for all values of $\lambda$, thus avoiding the singularity in $f(r)$ as desired.

\section{B. Simulations}

The evolution of the diffusion equation was simulated using Matlab ${ }^{\circledR}$ and the weighting function found in the previous subsection. The numerical scheme used for the

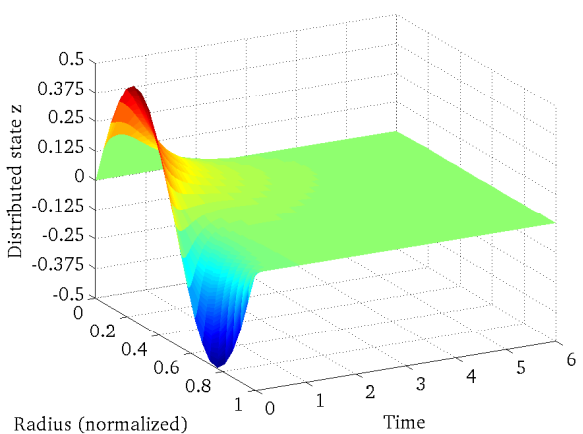

Fig. 5. Evolution of the state with no disturbance and minimum diffusivity.

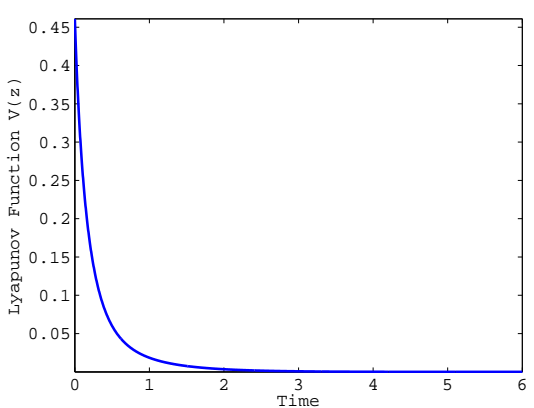

Fig. 6. Evolution of the Lyapunov function with no disturbance and minimum diffusivity.

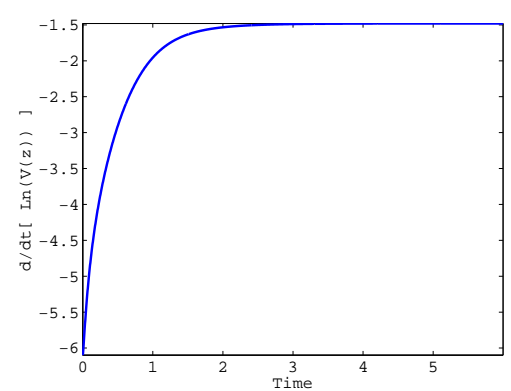

Fig. 7. Exponential convergence rate for $V(z)$ with no disturbance and minimum diffusivity.

simulations is an explicit finite-difference method with space and time steps chosen such that the CFL condition, $\max _{(r, t) \in[0,1] \times[0, T)}\{\eta\} \frac{\Delta t}{(\Delta x)^{2}} \leq 0.5$, is verified.

First, choosing the minimum values for the diffusion coefficients and without disturbances, the evolution of the distributed state can be seen in Figure 5. With this simulation, the evolution of the Lyapunov function and the equivalent rate of convergence are shown in Figures 6 and 7 , respectively. For this initial condition, the guaranteed rate of convergence is $\sim 23$ times smaller than the obtained one. Considering the fact that the condition imposed in Proposition 4 was verified at every point and that the diffusivity at the right boundary is $\sim 55$ times the one at the center, this level of conservatism is not unexpected.

Next, introducing a boundary disturbance $\varepsilon(t)=0.1+$ 


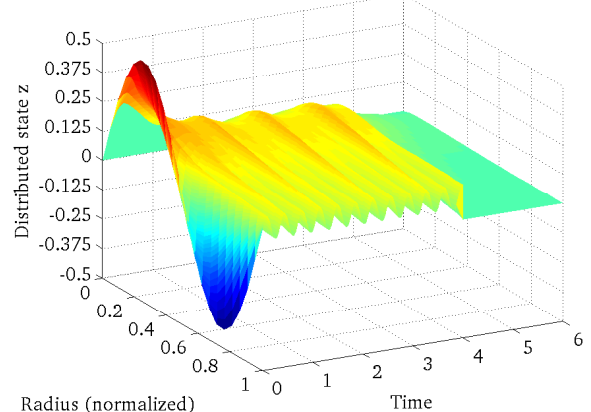

Fig. 8. Evolution of the state with disturbance acting until $t=4 \mathrm{~s}$ and time-varying diffusivity.

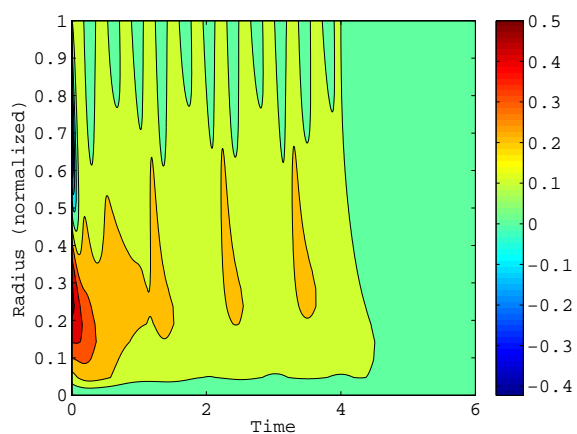

Fig. 9. Contour plot of the evolution of the state with disturbance acting until $t=4 \mathrm{~s}$ and time-varying diffusivity.

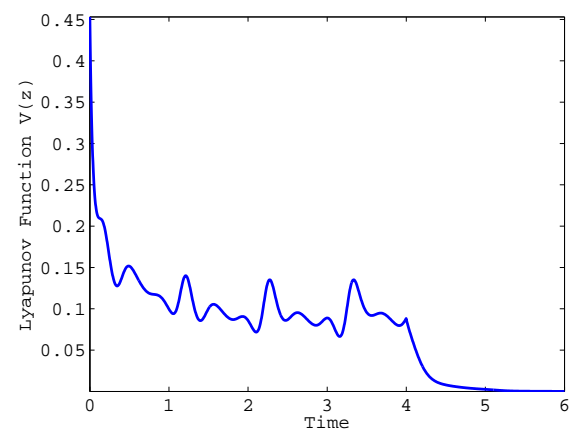

Fig. 10. Evolution of the Lyapunov function with disturbance acting until $t=4 \mathrm{~s}$ and time-varying diffusivity.

$0.05 \sin (4.56 \pi t)$ and letting the diffusivity coefficient vary with $a(t)=0.0107-0.0014 \cos (4 \pi t)$ and $\phi(r, t)=5.65+$ $1.65 \sin (2 \pi t)$ (independent of $r$ in this case). The resulting evolution can be seen in Figures 8 and 9. The behaviour of the Lyapunov function is shown in Figure 10. The Lyapunov function remains bounded when the disturbance is present and, once the disturbance is removed at $t=4 \mathrm{~s}$ the exponential convergence to zero is verified.

\section{Conclusions And Perspectives}

In this article, $\mathrm{D}^{1}$ ISS-like inequalities with respect to boundary disturbances are derived for a diffusive equation with singular coefficients stemming from a change between
Cartesian and polar coordinates. The $\mathrm{D}^{1}$ ISS condition is obtained by means of a strict Lyapunov function for the undisturbed system. Another contribution of this article is a detailed account of the method used to numerically find suitable weighting functions in order to implement the obtained results for some diffusivity profiles. Simulation results were obtained by discretizing the system using a finite-difference method.

Further works will tackle the problem of reducing the conservatism of this approach in order to better estimate the convergence rates, thus refining the $\mathrm{D}^{1}$ ISS inequalities for the system. Also, extensions to other forms of diffusivity profiles is desirable.

\section{ACKNOWLEDGEMENTS}

The authors are grateful to M. Krstic for stimulating discussions.

\section{REFERENCES}

[1] R. A. Baker and A. R. Bergen, Lyapunov stability and Lyapunov functions of infinite dimensional systems, IEEE Transactions on Automatic Control 14 (1969), no. 4, 325-334.

[2] F. Bribiesca Argomedo, C. Prieur, E. Witrant, and S. Brémond, A strict control Lyapunov function for a diffusion equation with time-varying distributed coefficients, Submitted for publication (2011).

[3] T. Cazenave and A. Haraux, An introduction to semilinear evolution equations, Oxford University Press, 1998.

[4] J.-M. Coron, G. Bastin, and B. d'Andréa Novel, Dissipative boundary conditions for one-dimensional nonlinear hyperbolic systems, SIAM Journal on Control and Optimization 47 (2008), no. 3, 1460-1498.

[5] J.-M. Coron and B. d'Andréa Novel, Stabilization of a rotating body beam without damping, IEEE Transactions on Automatic Control $\mathbf{4 3}$ (1998), no. 5, 608-618.

[6] J.-M. Coron, B. d'Andréa Novel, and G. Bastin, A strict Lyapunov function for boundary control of hyperbolic systems of conservation laws, IEEE Transactions on Automatic Control 52 (2007), no. 1, 2-11.

[7] B. Jayawardhana, H. Logemann, and E. P. Ryan, Infinite-dimensional feedback systems: the circle criterion and input-to-state stability, Communications in Information and Systems 8 (2008), no. 4, 413444.

[8] The circle criterion and input-to-state stability: New perspectives on a classical result, IEEE Control Systems Magazine 31 (2011), no. 4, 32-67.

[9] M. Krstic and A.T. Smyshlyaev, Adaptive boundary control for unstable parabolic PDEs-part I: Lyapunov design, IEEE Transactions on Automatic Control 53 (2008), no. 7, 1575-1591.

[10] W.J. Liu and M. Krstic, Backstepping boundary control of Burgers' equation with actuator dynamics, Systems \& Control Letters 41 (2000), 291-303.

[11] A. Lunardi, Analytic semigroups and optimal regularity in parabolic problems (H. Brezis, ed.), Progress in nonlinear differential equations and their applications, vol. 16, Birkhäuser, 1995.

[12] F. Mazenc and C. Prieur, Strict Lyapunov functions for semilinear parabolic partial differential equations, Mathematical Control and Related Fields 1 (2011), 231-250.

[13] A. Pazy, Semigroups of linear operators and applications to partial differential equations, Applied mathematical sciences, vol. 44, Springer Verlag New York, Inc., 1983.

[14] C. Prieur and F. Mazenc, ISS-Lyapunov functions for time-varying hyperbolic partial differential equations, Mathematics of Control, Signals, and Systems (to appear).

[15] A. Smyshlyaev and M. Krstic, Adaptive boundary control for unstable parabolic PDEs-part II: Estimation-based designs, Automatica 43 (2007), no. 9, 1543-1556.

[16] - Adaptive boundary control for unstable parabolic PDEs-part III: Output feedback examples with swapping identifiers, Automatica 43 (2007), no. 9, 1557-1564.

[17] E. D. Sontag, Input to state stability: Basic concepts and results, Nonlinear and optimal control theory, 2008, pp. 163-220. 